11:183-200.

Pasachoff, J.M. and Percy, J.R. (Eds.) 1990, The Teaching of Astronomy. Proceedings of the 105th Colloquium of the International Astronomical Union, Williamstown, MA. July 1988. Chapter 5: Computers, pp. 159-188. Cambridge University Press, Cambridge.

Szostak, R. 1990, "Two innovative devices for the teaching of astronomy" in Pasachoff and Percy (Eds.), The Teaching of Astronomy. Proceedings of the 105th Colloquium of the International Astronomical Union, Williamstown, MA. July 1998. pp. 218-222.

\title{
ASTRONOMY IN THE SCHOOLS: THE EUROPEAN SYSTEM
}

\author{
Lucienne Gouguenheim \\ Observatoire de Paris-Meudon, F-92195 Meudon, Cedex, France \\ E-mail: gouguenheim@obspm. fr
}

The following teaching activities and materials described hereafter, come from: (1) France, Comité de Liaison Enseignants et Astronomes (CLEA), (2) Germany, University of Münster (Roland Szostak, hereafter RS), (3) Spain, University of Catalogna Institut de Ciencias de l'Educacio (Rosa Maria Ros, ICE, (4) United Kingdom, Open University (OU) and (5) United Kingdom, a science centre in Northampton (Simply Space). Strikingly, these different groups suggest similar activities, based on observations and experiments aimed at studying the same concepts.

\section{ELEMENTARY SCHOOL AND JUNIOR HIGH SCHOOL}

1.1 Structure of space and time. A device which was invented by Roland Szostak is widely used now, both in Germany and France. A half-sphere made of glass or transparent plastic about $40 \mathrm{~cm}$ in diameter (it could be also a salad bowl) is set horizontally on a cardboard; the edge represents the horizon circle; right at the centre of the horizon circle, a small white paper is stuck which will be the target. The whole device is then set in a place that will be exposed to the Sun's light for the whole day and the meridian that was marked off on the plate will be aligned along the actual meridian using a compass. A small rectangular piece of cardboard with a small hole at the centre will be moved on the surface of the half-sphere in the approximate direction of the Sun. It casts a shadow on the ground of the model and a small luminous spot can be seen in the centre. The piece of cardboard must then be moved over the surface until the luminous spot falls right onto the target. The exact position of the hole will then be marked off with a felt-tip pen. The time of observation should also be recorded. In that way, several recordings of the Sun's position will be made at different times during the day. At the end of the day, all the points will 
be joined. The Sun's position at sunrise, at noon and at sunset will be thus determined.

Other recordings will be made on different dates. After a few days, the curves are distinctly different. The concept of season is evidenced by the varying length of the arcs drawn on the half-sphere.

CLEA suggest another activity using an almanac: the students list times of sunrise (R) and of sunset (S) in local time on each Sunday and plot them on a diagram; they study then the evolution of the length of daytime. They find on what date the daylight period is 12 hours long; they find the date and length of the longest and shortest day. The concept of seasons is evidenced here by the varying length of daytime. A school teacher has also made comparison with the mean value of maximum temperature. This activity together with RS half-sphere is published in Les Cahiers Clairaut special issue \#1, now available in French, in Polish and in English. CLEA publishes also a movable transparency illustrating the seasons.

RS invented a thermocoloric sphere: briefly, due to the painting which covers the sphere, the colour changes when the temperature increases. It is then possible to explain that the seasons are made possible because the axis of rotation of the Earthsphere is not perpendicular to the plane of its annual motion around the Sun. A similar activity is proposed by Simply Space, using terrestrial globes and an original experiment showing that the temperature of a tray of sand increases when the light rays become more vertical.

RS suggested another activity which is a by-product of the large sphere: He uses now a window oriented eastward. The target is a reference point on the wall in front of the window, and the path of the Sun is marked on the window each 5 minutes. Three days after, the route of the Sun is different. Now if one waits a year it is possible to measure the length of the year.

That the Sun does not rise or set every day in the same direction can be inferred from direct observations or using slides. Similar activities are described either by ICE and by CLEA (a set of 20 slides showing in particular the sunrise on the 21 st of each month. Then a panoramic view can be obtained from the slides, and offers the possibility of measuring the deviation of sunrise from the true East point. It is also possible to determine the obliquity of the ecliptic. The slides are available in French, English and Spanish, and ICE activity file both in Spanish and English.

1.2 Moon phases. Because most of the children have in mind that they are due to the fact that the Moon goes through the shadow of the Earth, different devices have been made in order to show the phases of a sphere lighted by the Sun or by a light which mimics the Sun. This was done by Simply Space using a round cake box with 30 holes around it. CLEA publishes slides showing simultaneously the phases of the Moon and the phases of a ball. They are available in French, English and Spanish. CLEA publishes also an ensemble of activity files available in French and in English.

\section{SENIOR HIGH SCHOOLS}

2.1 Relative motion. A classical activity relies on the observation of sunspots; CLEA publishes a set of 20 slides, available both in French and in English which mimics the observation of the Sun, from which can be derived first the synodic and second the sideral period of rotation of the Sun, taking account of Earth's motion around the 
Sun.

CLEA publishes also slides showing the retrograde motion of Mars in the Pleiades from which both the apparent motion and the variation of apparent magnitude of Mars can be determined using the Hyades and Pleiades stars as references.

The solar time at which a given apparent position of stars can be observed results from the two different motions of the Earth: its diurnal rotation and its annual revolution. The combination of these 2 motions can be illustrated by constructing either an astrolabe (ICE and CLEA) or a starfinder.

2.2 Analysis of light. In French senior high schools, a device initiated here in Holland by Minnaert is now widely used for measuring the solar constant.

RMR (ICE) published slides of variable stars (delta Cephei and Algol) from which students can determine very good light curves. Spectra of the Sun and stars are also widely used for illustrating either the Doppler effect or chemical composition. As an example, the spectrum of Beta Orionis, flanked by two emission spectra of argon, was taken by French school teachers using the $60 \mathrm{~cm}$ telescope at Pic du Midi, which is now open to amateurs. It is possible to ask the students to calibrate the spectrum, using the argon spectrum, from which they determine quickly the most intense features at $696.5 \mathrm{~nm}$ and around 420 . They are asked then to determine the wavelengths of 12 absorption lines and to identify them from a table.

\title{
CURRICULUM, ACTIVITIES AND RESOURCES: THE NORTH AMERICAN SYSTEM
}

\author{
John R. Percy
}

Erindale Campus, University of Toronto, Mississauga, Ontario, Canada L5L 1C6

E-mail: percy@astro.utoronto.ca

Introduction. The difference between the European and North American systems of education has been eloquently described by Wentzel (1990); see also other papers in the proceedings of IAU Colloquium \#105, the triennial reports of Canada, Mexico and the US in IAU Commission 46's newsletter, and an ongoing series of short papers on astronomy education in Mercury, published by the Astronomical Society of the Pacific, dealing with astronomy education in general $(22, \# 2)$, planetarium education $(22, \# 5)$, an astronomy camp $(22, \# 6)$, gender issues $(23, \# 1)$, image processing (23, \#2), student misconceptions $(23, \# 3)$ and education at the HarvardSmithsonian Center for Astrophysics (23, \#4).

Current concerns in North America include: a decline in students' interest and ability in science, low prestige and rewards of teaching as a profession, and lack of science and math background among elementary school teachers (a universal concern!). These concerns must be viewed in the context of the deepening gulf 